\title{
The tumor suppressor RASSF1A is a novel effector of small $G$ protein Rap1A
}

\author{
Sunil K. Verma ${ }^{1,2,3,5}$, Trivadi S. Ganesan ${ }^{1,2,6}$, Uday Kishore ${ }^{7}$, Peter J. Parker ${ }^{3,4} \bowtie$ \\ 1 Department of Medical Oncology, Medical Sciences Division, The University of Oxford, Oxford OX3 9DU, UK \\ 2 Ovarian Cancer Group, CRUK Molecular Oncology Laboratories, Weatherall Institute of Molecular Medicine, John Radcliffe \\ Hospital, Headington, Oxford OX3 9DS, UK \\ 3 Protein Phosphorylation Laboratory, London Research Institute, CRUK Lincoln's Inn Fields Laboratories, 44 Lincoln's Inn \\ Fields, London WC2A 3PX, UK \\ ${ }^{4}$ Kings College London, The Division of Cancer Studies, Section of Cancer Cell Biology and Imaging, 2nd Floor, New Hunt's \\ House, Guy's Hospital, St Thomas Street, London SE1 1UL, UK \\ ${ }^{5}$ Current address: Center for Cellular and Molecular Biology, Uppal Road, Hyderabad 500 007, India \\ ${ }^{6}$ Current address: Cancer Institute \& Institute of Molecular Medicine, Amrita Institute of Medical Sciences, Elamakkara P.O. \\ Kochi, Kerala 682 026, India \\ 7 Center for Infection, Immunity and Disease Mechanisms, Biosciences, School of Health Sciences and Social Care, Brunel \\ University, Uxbridge UB8 3PH, West London, UK \\ $\bowtie$ Correspondence: peter.parker@cancer.org.uk
}

Received February 21, 2011 Accepted March 13, 2011

\section{ABSTRACT}

Rap1A is a small $G$ protein implicated in a spectrum of biological processes such as cell proliferation, adhesion, differentiation, and embryogenesis. The downstream effectors through which Rap1A mediates its diverse effects are largely unknown. Here we show that Rap1A, but not the related small $G$ proteins Rap2 or Ras, binds the tumor suppressor Ras association domain family $1 \mathrm{~A}$ (RASSF1A) in a manner that is regulated by phosphorylation of RASSF1A. Interaction with Rap1A is shown to influence the effect of RASSF1A on microtubule behavior.

KEYWORDS RASSF1A, Rap1A, microtubule, vimentin, protein-protein interaction

\section{INTRODUCTION}

Rap1A (Krev-1) is a member of the Ras superfamily of small GTP-binding proteins and has highest homology to Ras. Rap1A behaves as an antagonist of oncogenic K-Ras activity in NIH3T3 cells (Kitayama et al., 1989) and of polyoma middle-T antigen in Rat-2 cells (Jelinek and Hassell, 1992). It inhibits transformation of human prostate cancer cells (Burney et al., 1994), hamster pancreatic adenocarcinoma cells (Leach et al., 1998) and of ethylcarbamate induced lung adenomas in transgenic mice (Damak et al., 1996). However, Rap1A does not prevent Ras induced transformation in human HT1080, EJ30 or SW48 cells (Sato et al., 1994). Rap1A also plays a critical role in the regulation of normal morphogenesis in the eye disk and the ovary during embryo development (Hariharan et al., 1991; Asha et al., 1999). Recently, Rap1A has been implicated in integrin-mediated adhesion through RapL (Katagiri et al., 2003) and modulation of the actin cytoskeleton through RIAM (Lafuente et al., 2004). Beyond these targets, the downstream effectors through which Rap1A mediates its diverse effects are largely unknown.

The Ras association domain family $1 \mathrm{~A}$ (RASSF1A) gene is localized at chromosome region 3p21.3, which frequently undergoes loss of heterozygosity in human solid tumors due to promoter methylation (Sekido et al., 1998; Dammann et al., 2000; Lerman and Minna, 2000). RASSF1A contains an amino-terminal cysteine-rich region, similar to the diacylglycerol-binding domain ( $\mathrm{C} 1$ domain) found in the protein kinase $C$ family of proteins and a carboxy-terminal Ras-association (RA) domain (Sekido et al., 1998; Dammann et al., 2000; Lerman and Minna, 2000; Dammann et al., 2005). The functional analysis of RASSF1A reveals its involvement in apoptotic signalling (Vos et al., 2000), microtubule stabilization (Liu et al., 2003; Dallol et al., 2004; Rong et al., 2004) and 


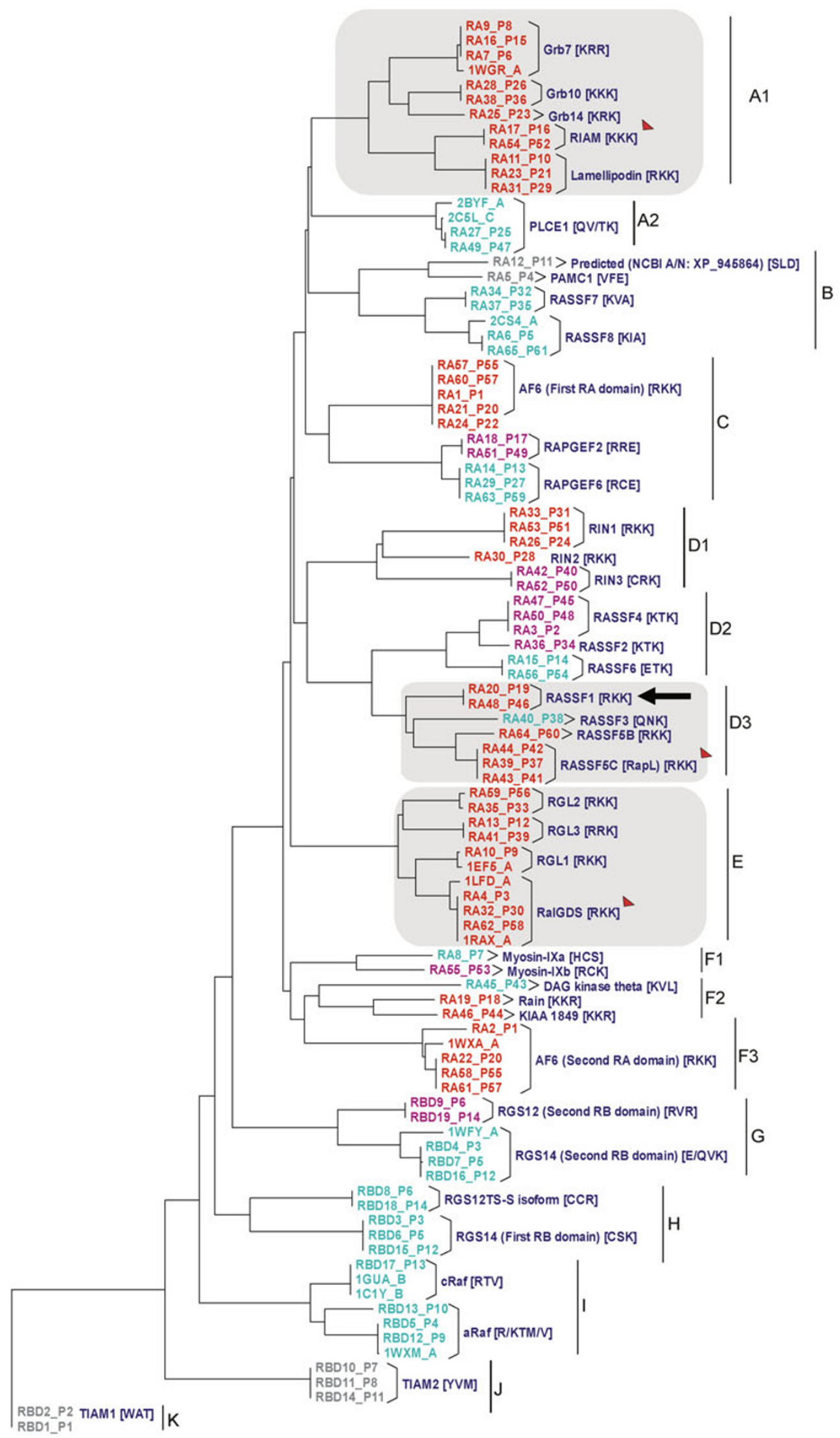


G1-S progression (Shivakumar et al., 2002). RASSF1A gene knock-out mice are highly susceptible to various tumors (Tommasi et al., 2005). RASSF1A thus represents an epigenetically inactivated tumor suppressor in human carcinogenesis (Dammann et al., 2005).

In this study, we report that RASSF1A is a novel effector of Rap1A, linking Rap1A to microtubules. We also show specific and regulated interaction of RASSF1A with ectopically expressed activated Rap1A. This interaction appears to have robust effects on the intracellular distribution of the microtubule network.

\section{RESULTS}

\section{Identification of RASSF1A as a Rap1A-interacting protein}

X-ray crystallographic studies have shown that the highly acidic effector residues in the switch I region of Rap1A interact with the positively charged patch generated by the critical basic residues located in strands $\beta 1, \beta 2$, and the end of $\alpha 1$ helix of RB/RA domain of the proteins $\mathrm{CRaf}$ and RalGDS (Nassar et al., 1995; Nassar et al., 1996; Huang et al., 1997). Furthermore, it has recently been shown that the novel effector RapL interacts with Rap1A through its RA domain and that the same critical basic residues within the RA domain of RapL play a key role in this interaction (Katagiri et al., 2003). Conserved domain neighborhood analysis performed on $84 \mathrm{RA} / \mathrm{RB}$ domains from 75 human protein entries of the SMART database (Table S1) identified RASSF1A as the most homologous domain neighbor of the RA domain of RapL (Fig. 1). Structural comparisons of the 3D models of RA domain of these proteins further suggested that the structure of the RA domain of RASSF1 was remarkably similar to the RA domain of RapL as indicated by a Ca r.m.s.d (root mean square deviation) of $1.05 \AA$ (Fig. 2A). The 3D structure-based primary amino acid alignment indicated that the specific basic residues in $\beta 1, \beta 2$ and $\alpha 1$ strands known to be critical for interaction of Rap1A with its effectors (indicated as red arrows in Fig. 2A) (Nassar et al., 1995; Nassar et al., 1996; Huang et al., 1997) were highly conserved in RASSF1A.

In vitro protein association assays demonstrated that FlagRASSF1A specifically interacted with GST-Rap1A (Fig. 2B). To further assess the dependence of RASSF1A-Rap1A interaction on the activation state of Rap1A, the GSTRap1A was loaded with either GDP- $\beta$-S or GTP-y-S and binding reactions performed. It was found that FlagRASSF1A preferentially interacted with GTP-Rap1A (Fig. $2 C$ ), suggesting that RASSF1A is a novel interactor of activated Rap1A.

\section{Rap1A-RASSF1A interaction is regulated by phosphorylation of RASSF1A}

In order to investigate whether RASSF1A phosphorylation could be induced in this model, 293T cells transfected with Flag-RASSF1A were treated with the cell permeable phosphatase inhibitor calyculin A. Cell lysates were prepared, resolved by SDS-PAGE and subjected to immunoblotting (IB) with RASSF1A antibody. Calyculin A treatment resulted in retardation of the electrophoretic mobility of RASSF1A on the SDS-PAGE (Fig. 3A, upper panel), indicative of the phosphorylation state of RASSF1A. Interestingly, calyculin A treatment also resulted in loss of Rap1A-RASSF1A interaction in vitro (Fig. $3 \mathrm{~A}$, middle panel), suggesting a RASSF1A phosphorylation dependent regulation of Rap1A-RASSF1A interaction. This dependence on the phosphorylation state of RASSF1A is a further validation of the specificity of this interaction.

Several putative phosphorylation sites were predicted by in silico analysis within RASSF1A; the sites S197 and S203, which are located within the RA domain of RASSF1A, have been established as novel protein kinase $C$ (PKC) phosphorylation sites (Verma et al., 2008). In order to assess whether the specific phosphorylation of these two sites could modulate the interaction of RASSF1A with activated Rap1A, the 293T cells transfected with wild type or S197,

Figure 1. Neighbor Joining (NJ) tree showing the domain neighbors of RapL-RA, RIAM-RA and RaIGDS-RA. The dendrogram is based on the multiple sequence alignments prepared by superimposing the primary amino acid sequences of 84 RA/ RB domain entries of SMART (Table S1) on the alignment profile of 12 different high resolution 3D structures of RA/RB domains superimposed by Combinatorial Extension (CE) algorithm (Shindyalov and Bourne, 1998). The PDB structures used to generate the alignment profile were 1RAX:A (Solution structure of RalGDS-RA), 1LFD:A (Crystal structure of RalGDS), 1EF5:A (Solution structure of RGL), 1WXA:A (Solution structure of AF6-RA2), 1WGR:A (Solution structure of Grb7), 1GUA:B (Crystal structure of cRaf), 1C1Y:B (Crystal structure of cRaf), 2CS4:A (Solution structure of C12orf2), 1WXM:A (Solution structure of aRaf), 1WFY:A (Solution structure of RGS14-RA2) and 2BYF:A (solution structure of PLC-epsilon RA2). The codes for the color are as follows Red: all the three critical residues are conserved (Identical or similar); Pink: 2 residues conserved; Green: 1 residue conserved; Gray: None of the critical residues are conserved; Blue: The proteins to which the RA/RB domain belongs. Consensus of critical residues at three positions is noted in brackets with the name of the protein. The proteins flagged with red arrows are the proteins that have an RA/RB domain that is known to interact with Rap1A as published before in the literature [RIAM: (Lafuente et al., 2004); RapL (Franke et al., 1997); (Katagiri et al., 2003)]. Clusters of domains (Cluster A-K) inferred from the tree are indicated. Three shaded blocks of clusters (Cluster A1, D3 and E) are the clusters of RA/RB domains that include at least one RA/RB domain which is known to interact with Rap1A. Protein shown with black arrow was selected as a candidate protein to further assess as a novel effector of Rap1A. 
A

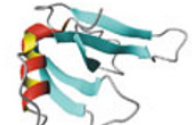

RapL

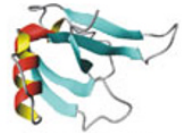

RASSF1A

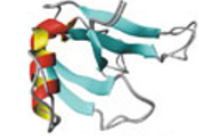

RapL/RASSF1A
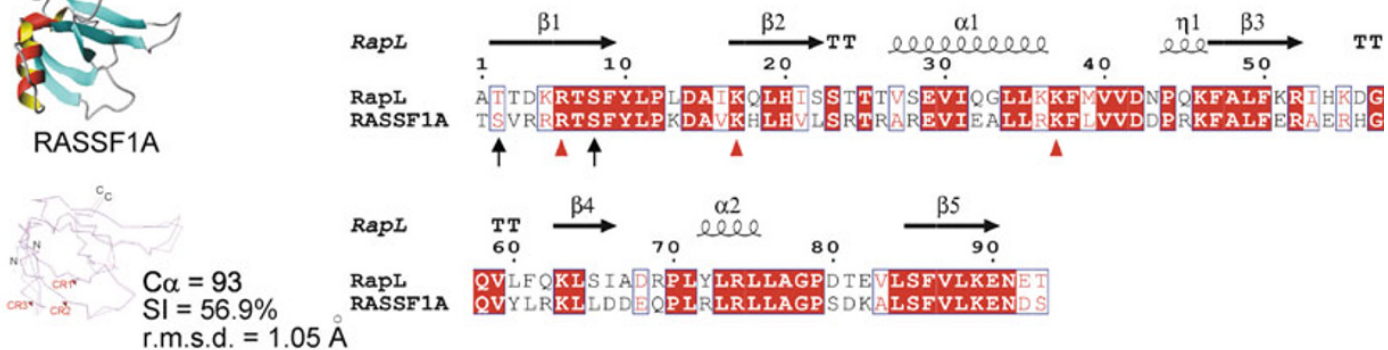

B

Untransfected Flag-RASSF1A

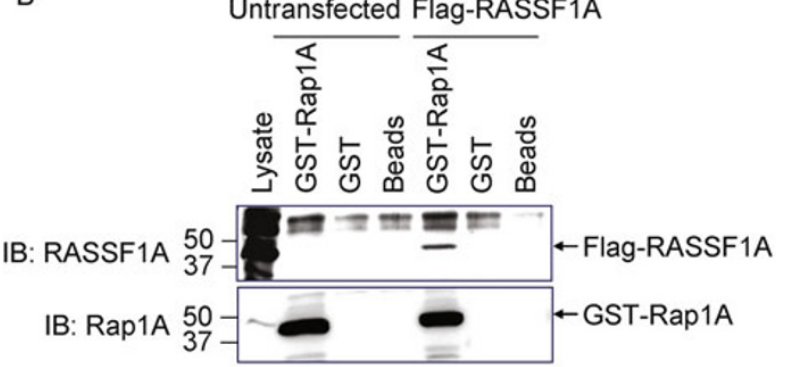

C

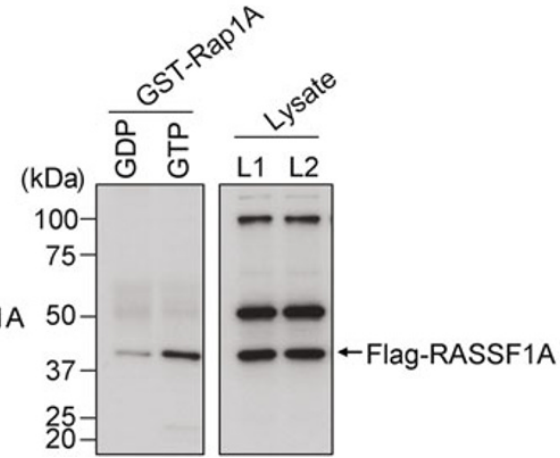

Figure 2. RASSF1A interacts with GTP-Rap1A. (A) 3D models, Ca superimpositions, sequence alignment and secondary structure assignments for the RA domains of RapL and RASSF1. A 3D model of the RA domains of RapL and RASSF1A was generated using 1RAX:A (RalGDS-RA) as a template in alignment mode of SWISS-MODEL. Ca Superimposition of these models as indicated was generated using the Combinatorial Extension (CE) algorithm (Shindyalov and Bourne, 1998). All the models were drawn with MOLMOL (Koradi et al., 1996). Wire rendering of the superimposition was drawn with Cn3D (Hogue, 1997). The number of aligned Ca residues, \% sequence identity $(\mathrm{SI})$ and r.m.s. deviations for $\mathrm{Ca}$ traces are shown. Numbering at the top corresponds to the amino acid sequence of full length RapL-RA domain delineated from human RapL, Swiss-Prot accession number Q8WWW0. Letters marked with red arrows correspond to three critical residues in the RalGDS-RA domain (Huang et al., 1997). Positions of these residues in $3 \mathrm{D}$ models are shown with red arrows in the wire rendering as well as in the alignment shown. The specific serine residues marked with black arrows are the PKC phosphorylation sites in RASSF1A (Verma et al., 2008). (B) RASSF1A interacts with Rap1A in vitro. HEK293 cells were transfected with or without the pCDNA3-Flag-RASSF1A expression vector (MATERIALS AND METHODS). At $24 \mathrm{~h}$ after transfection, the cells were harvested and lysed; equal amounts of cell lysates from untransfected or FlagRASSF1A transfected cells as indicated were subjected to pull-down assay using Glutathion Sepharose Transferase (GST) fusion protein for Rap1A (GST-Rap1A) and subjected to immunoblot (IB) analysis using antibodies as indicated in the figure legend. (C) RASSF1A preferentially interacts with activated Rap1A in vitro. Glutathion Sepharose Transferase (GST) fusion protein for Rap1A (GST-Rap1A) in crude bacterial lysates was prepared and loaded with either GDP- $\beta$-S or with GTP-y-S following the procedure described in MATERIALS AND METHODS. The lysate from Flag-RASSF1A transfected HEK293 cells was equally divided into two halves and each half was subjected to pull-down with the nucleotide loaded Rap1A as indicated. Bound protein was probed by western blotting for RASSF1A. Lanes L1 and L2 are the input lysates from Flag-RASSF1A transfected HEK293 cell used for pulldown by GDP or GTP loaded GST-Rap1A, respectively. These results are representative of 2 separate experiments.

203A double mutant (i.e., which cannot be phosphorylated on these specific sites) of Flag-RASSF1A were treated with phorbol 12-myristate 13-acetate (PMA) + calyculin A and the cell lysates prepared from these cells were employed to assess the interaction of RASSF1A with activated Rap1A by in vitro pull-down assay. The PMA + calyculin A induced phosphorylation of RASSF1A at S197 and S203 was monitored by phospho-RASSF1A S197 and phosphoRASSF1A S203 specific immune sera PPA545 and PPA548, respectively. The specificity of these phosphospecific antisera to the RASSF1A phosphorylation sites has been documented elsewhere (Verma et al., 2008). As expected, the wild type RASSF1A, but not the AA mutant, was phosphorylated at these sites on PMA + calyculin A treatment (Fig. 3B). The in vitro GTP-Rap1A pull-down assay performed on these lysates demonstrated that upon PMA + calyculin A treatment, the wild type RASSF1A lost its interaction with activated Rap1A (Fig. 3C). It was also found that the AA mutant of RASSF1A, which is not phosphorylated at $\mathrm{S} 197$ and $\mathrm{S} 203$ in response to PMA + calyculin A treatment, also lost its interaction with activated Rap1A (Fig. $3 C$ ), indicating that RASSF1A phosphorylation at S197 and S203 was not responsible for regulating interaction with Rap1A. The specific phosphorylation site(s) within 
A

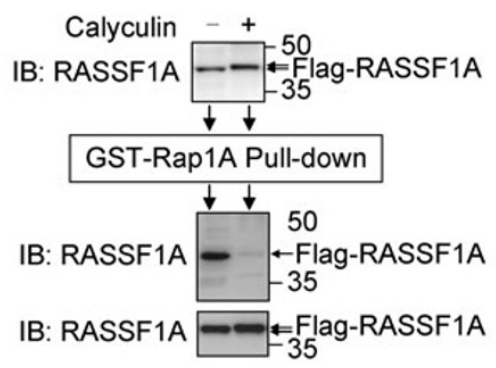

B

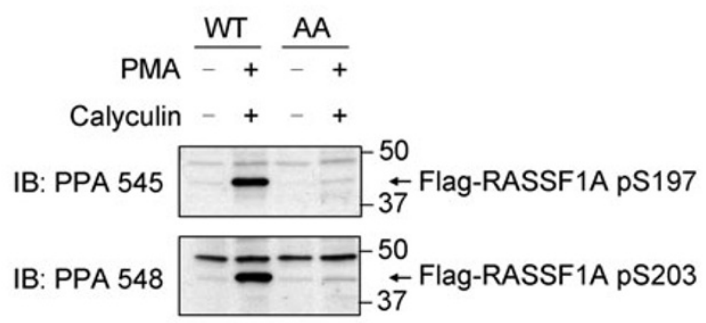

C

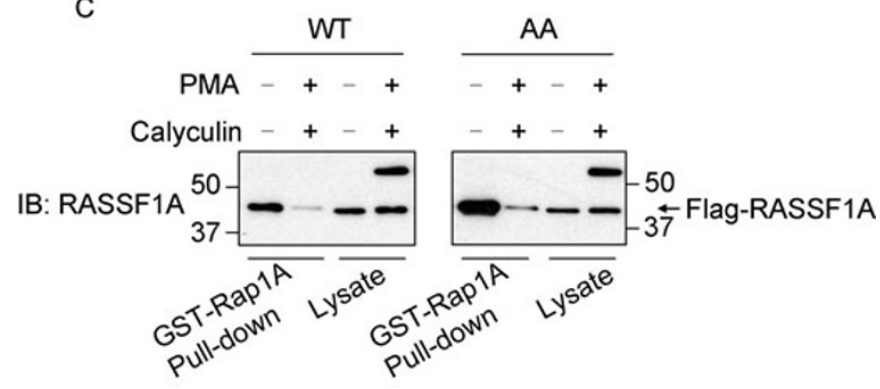

Figure 3. Rap1A-RASSF1A interaction is regulated by phosphorylation of RASSF1A. (A) Retardation of the electrophoretic mobility of RASSF1A upon calyculin A treatment and influence on Rap1A-RASSF1A interaction. 293T cells grown to $90 \%$ confluence were transfected with the Flag-RASSF1A. At $24 \mathrm{~h}$ after transfection, the cells were treated with or without $100 \mathrm{nmol} / \mathrm{L}$ calyculin A for $30 \mathrm{~min}$. A small fraction of the cell lysates prepared was resolved by SDS-PAGE and subjected to immunoblotting (IB) with RASSF1A antibody (upper panel). The rest of the lysates from calyculin A treated or untreated cells was subjected to pull-down assay using GTP loaded GST-Rap1A as probe. For this, the above lysate was incubated for 90 min with GTP loaded GST-Rap1A precoupled with glutathione Sepharose 4B (GSH) beads followed by 3 washes with MLB buffer. The protein bound to GSH beads was extracted with $2 \times$ NuPAGE $^{\circledR}$ SDS Sample Buffer and probed by western blotting for RASSF1A (middle panel). The input lysates are shown in the lower panel. (B) 293T cells grown to $90 \%$ confluence were transfected with the wild type Flag-tagged RASSF1A (WT) or with the AA double mutant of Flag-tagged RASSF1A. At $24 \mathrm{~h}$ after transfection, the cells were stimulated or not with $0.8 \mu \mathrm{mol} / \mathrm{L}$ PMA + $100 \mathrm{nmol} / \mathrm{L}$ calyculin A for $30 \mathrm{~min}$ followed by lysis in MLB buffer. The lysates were immunoblotted with Phospho-RASSF1A S197 (upper panel) and Phospho-RASSF1A S203 (lower panel) specific immune sera as indicated. (C) The lysates obtained in - B were incubated with GTP loaded GST-Rap1A precoupled with glutathione Sepharose 4B (GSH) beads for 90 min followed by $3 \times$ washing with MLB buffer. The protein bound to GSH beads was extracted with $2 \times$ NuPAGE $^{\circledR}$ SDS Sample Buffer and loaded in gel along with input lysate followed by IB with RASSF1A antibody. These results are representative of 2 separate experiments.

RASSF1A, which are implicated in regulation of its interaction with GTP-Rap1A, remain to be identified.

\section{RASSF1A interacts with Rap1A on a circular perinuclear network}

Having established that RASSF1A can interact with activated Rap1A in a regulated manner, the subcellular localization of ectopically expressed EGFP-Rap1A and Flag-RASSF1A was examined by confocal microscopy in transfected Cos-7 cells, which do not express endogenous RASSF1A. It was not possible to perform these experiments on the endogenous Rap1A since no antibody suitable for immunofluorescence was available. As suggested before (Liu et al., 2003; Dallol et al., 2004; Rong et al., 2004; Song et al., 2004), FlagRASSF1A was present as a circular network in the cytoplasm and perinuclear region in Cos-7 cells (Fig. 4A), while the EGFP-Rap1A expressed alone was predominantly present in 
punctate structures in the perinuclear region of these cells (Fig. 4A). Similar subcellular localization of endogenous Rap1A in BHK cells and in NIH3T3 cells (Pizon et al., 1994) or ectopically expressed Rap1A in Cos-1 has previously been demonstrated (Mochizuki et al., 2001). Interestingly, the coexpression of EGFP-Rap1A with Flag-RASSF1A led to a robust redistribution of EGFP-Rap1A from the punctate perinuclear structures to the circular perinuclear network where the EGFP-Rap1A and Flag-RASSF1A became strongly co-localized (Fig. 4A), indicating interaction of these proteins within cells.

It is known that Rap1A, Rap2 and Ras share a $100 \%$ identical core effector region (see Bos et al., 2001). It is possible therefore that these proteins share some of their effectors. Indeed, it has been shown that many of the Ras effectors such as CRaf, AF6 and RalGDS are shared by Rap1A (though with differential affinities) (Herrmann et al., 1996; Vetter et al., 1999; Boettner et al., 2000; Zhang et al., 2005). In order to test whether RASSF1A is a specific effector of Rap1A compared to Rap2 and Ras, the subcellular localization of Flag-RASSF1A in Cos-7 cells expressing EGFP-Rap2 or EGFP-Ras was examined by confocal microscopy. Unlike EGFP-Rap1A, which is redistributed from punctate perinuclear structures to the circular perinuclear network along with Flag-RASSF1A, EGFP-Rap2 or EGFP-Ras was neither redistributed nor co-localized with Flag-RASSF1A (Fig. 4A). These observations were further substantiated by examining the in vitro association of FlagRASSF1A with the GTP-Y-S loaded GST-Rap1A, GST-Rap2 and GST-Ras proteins. It was found that RASSF1A selectively interacted with GTP-Rap1A. No binding was detected with either GST beads alone (negative control), GTP-Rap2 or GTP-Ras (Fig. 4B), suggesting that RASSF1A was a specific effector of Rap1A and it was not shared either by Rap2 or Ras. Together, these results demonstrate that RASSF1A selectively interacts with activated Rap1A in vitro and in vivo; and ectopically expressed Flag-RASSF1A and EGFP-Rap1A co-localize on a circular perinuclear network in Cos-7 cells.

\section{Rap1A-RASSF1A interaction plays a role in the intracellular distribution of microtubules}

Previous studies have shown that RASSF1A associates with microtubules in the interphase cells (Liu et al., 2003; Dallol et al., 2004; Rong et al., 2004; Song et al., 2004). In order to confirm this finding, Cos-7 cells transfected with Flag-tagged RASSF1A were immunostained for Flag-RASSF1A and $\alpha-$ tubulin, and analyzed by confocal microscopy. It was found that RASSF1A indeed co-localized with microtubules (Fig. 5). In addition, it was observed that ectopic expression of RASSF1A in Cos-7 cells appeared to cause rearrangement of the microtubules into circular rings, whereas the untransfected or vector transfected cells showed the microtubules
A

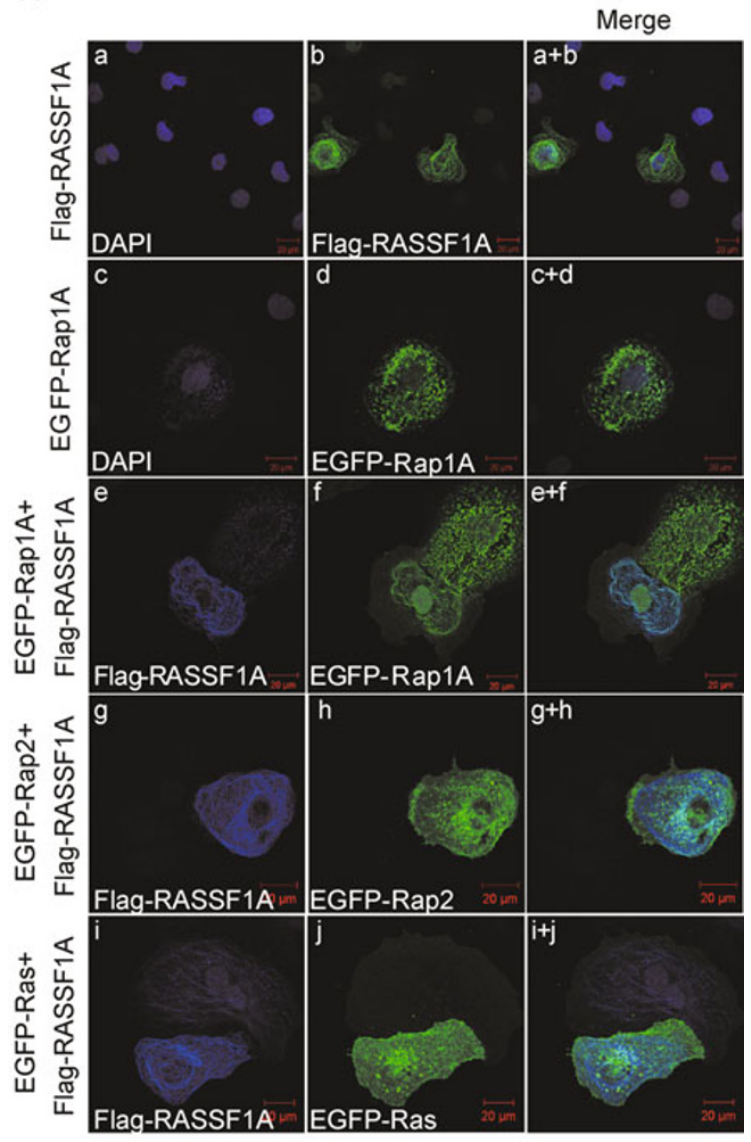

B

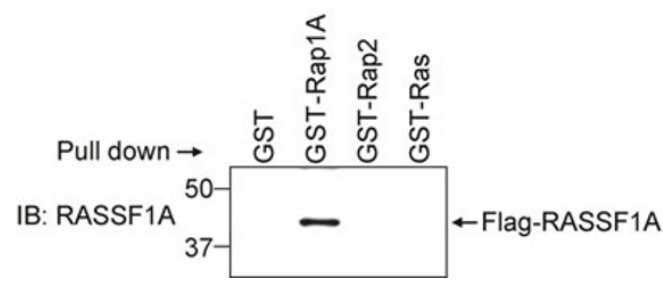

Figure 4. RASSF1A is a specific effector of Rap1A. (A) Cos-7 cells transfected with the indicated vector (corresponding to legend on the right side of each panel) were immunostained using specific antibody indicated inside the panels and analyzed using confocal microscopy. FlagRASSF1A was visualized using Flag-M1 antibody (Rabbit polyclonal, Sigma-Aldrich). Nuclei are counterstained with DAPI. Two-color superimposed images (Merge) of each panel are shown as indicated. The scale bar is equivalent to $20 \mu \mathrm{m}$. (B) The crude bacterial lysates containing GST-Rap1A, GST-Rap2 or GST-Ras proteins were treated with GTP-Y-S following the procedure described in MATERIALS AND METHODS. Equal amounts of Flag-RASSF1A transfected Hek293 cell lysate were incubated with a GTP-y-S loaded, active forms of GST-Rap1A, Rap2 or Ras pre-bound to glutathione Sepharose 4B (GSH) beads in MLB buffer; the $\mathrm{GSH}$ beads alone served as a negative control. Following 90 min incubation at $4^{\circ} \mathrm{C}$, beads were pelleted and washed three times with ice-cold MLB buffer. Protein was eluted from the beads in $2 \times$ Laemmli buffer and probed by western blotting for RASSF1A as indicated. 
radiating from a microtubule-organizing center (MTOC) (Bailly and Bornens, 1992) (Fig. 5). This phenotype is consistent with microtubule staining in cell lines with or without endogenous RASSF1A expression as suggested before (Dallol et al., 2004). Together, this data confirms that RASSF1A is a microtubule associating protein and has an effect on the intracellular distribution of the microtubule network. Since RASSF1A was found here as a Rap1A-interacting protein, we further sought to investigate the effects of Rap1A on the distribution pattern of microtubules. To explore this, EGFPRap1A transfected Cos-7 cells were immunostained for $\alpha$ tubulin. It was found that similar to RASSF1A, ectopic expression of EGFP-Rap1A also induced microtubule circularization in Cos-7 cells and loss of the MTOC (Fig. 5).
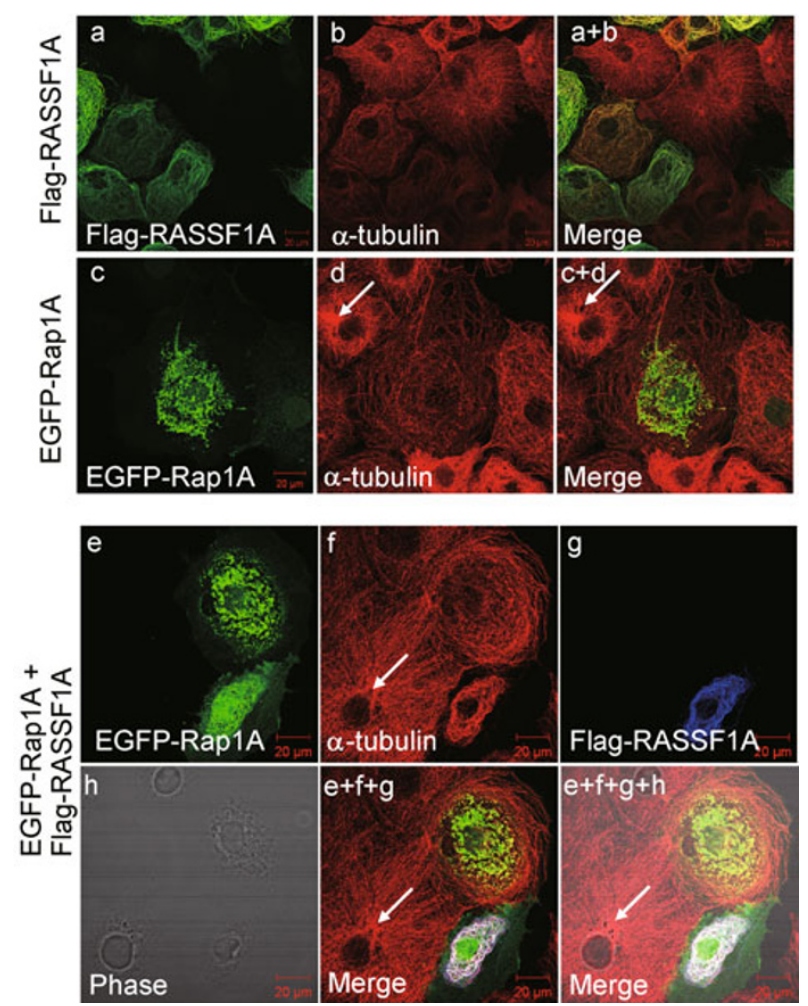

Figure 5. Co-localization of RASSF1A and Rap1A and their effect on the microtubule network. Cos-7 cells transfected with the indicated vector (corresponding to legend on the right side of each panel) were immunostained using specific antibody indicated inside the panels and analyzed using confocal microscopy. Flag-RASSF1A was visualized by Flag-M1 antibody and endogenous microtubules by the $\alpha$-tubulin antibody. Microtubules (red) radiating from a microtubule-organizing center (MTOC) (white arrows) are shown. Two-color/multi-color superimposed images (Merge) of each panel are shown as indicated. MTOC (wherever is indicated with white arrows). Each panel represents a mixture of transfected and non-transfected cells. The scale bar is equivalent to $20 \mu \mathrm{m}$.
Given that Rap1A and RASSF1A individually could modulate the intracellular distribution of the microtubules and that RASSF1A interacts with Rap1A in vitro and in vivo, the effect of the Rap1A-RASSF1A association on the intracellular distribution of the microtubule network was investigated. Cos-7 cells transiently transfected with the expression vectors carrying EGFP-Rap1A and Flag-tagged RASSF1A were co-stained for Flag-tag and $\alpha$-tubulin to detect RASSF1A and microtubules, respectively. Fig. $5(\mathrm{e}-\mathrm{h})$ shows a field of cells representing different populations of Cos-7 cells including: untransfected cells, EGFP-Rap1A transfected cells, and cells that are co-transfected with EGFP-Rap1A + Flag-RASSF1A. As expected, the untransfected or vector transfected cells exhibited a normal pattern of microtubules with radial arrays emanating from the MTOC and extending towards the cell periphery (Fig. 5). In the FlagRASSF1A transfected Cos-7 cells the microtubule network was circularized. Interestingly, the circularized microtubule network was strongly retracted around the nucleus in the cells that express both, the EGFP-Rap1A and Flag-RASSF1A, leaving much of the cell body devoid of a microtubule network (Fig. 5). Taken together, this data suggests that in Cos-7 cells ectopically expressed Rap1A or RASSF1A leads to the circularization of the microtubule network and that coexpression of both Rap1A and RASSF1A induces bundling of circularized microtubules around the nucleus where the Rap1A-RASSF1A complex becomes co-localized.

It has been shown that RASSF1A has microtubule stabilizing properties (Shivakumar et al., 2002; Liu et al., 2003; Dallol et al., 2004; Rong et al., 2004). This was further confirmed here by confocal microscopy of Flag-RASSF1A transfected, nocodazole treated Cos-7 cells along with appropriate controls. Nocodazole is a microtubule-specific agent that binds to the subunits of microtubules and prevents their polymerization (Downing, 2000). Treatment of Cos-7 cells with nocodazole caused dispersal of microtubules in empty vector transfected or untransfected cells (Fig. 6). However, in Flag-RASSF1A-expressing cells, microtubules still maintained their modified network structure (Fig. 6). FlagRASSF1A and EGFP-Rap1A cotransfected, nocodazole treated Cos-7 cells displayed an intact but retracted perinuclear-network of microtubules, where Flag-RASSF1A and EGFP-Rap1A co-localized (Fig. 6). Thus, RASSF1A stabilizes microtubules and on interaction with Rap1A, this stabilization is retained albeit in an altered, nuclear retracted form.

\section{RASSF1A and Rap1A influence the cellular processes that are directly or indirectly dependent on intracellular microtubule organization}

The microtubule network plays an important role in the maintenance of cellular structures including the microfilament network (Liao and Gundersen, 1998; Prahlad et al., 1998; 

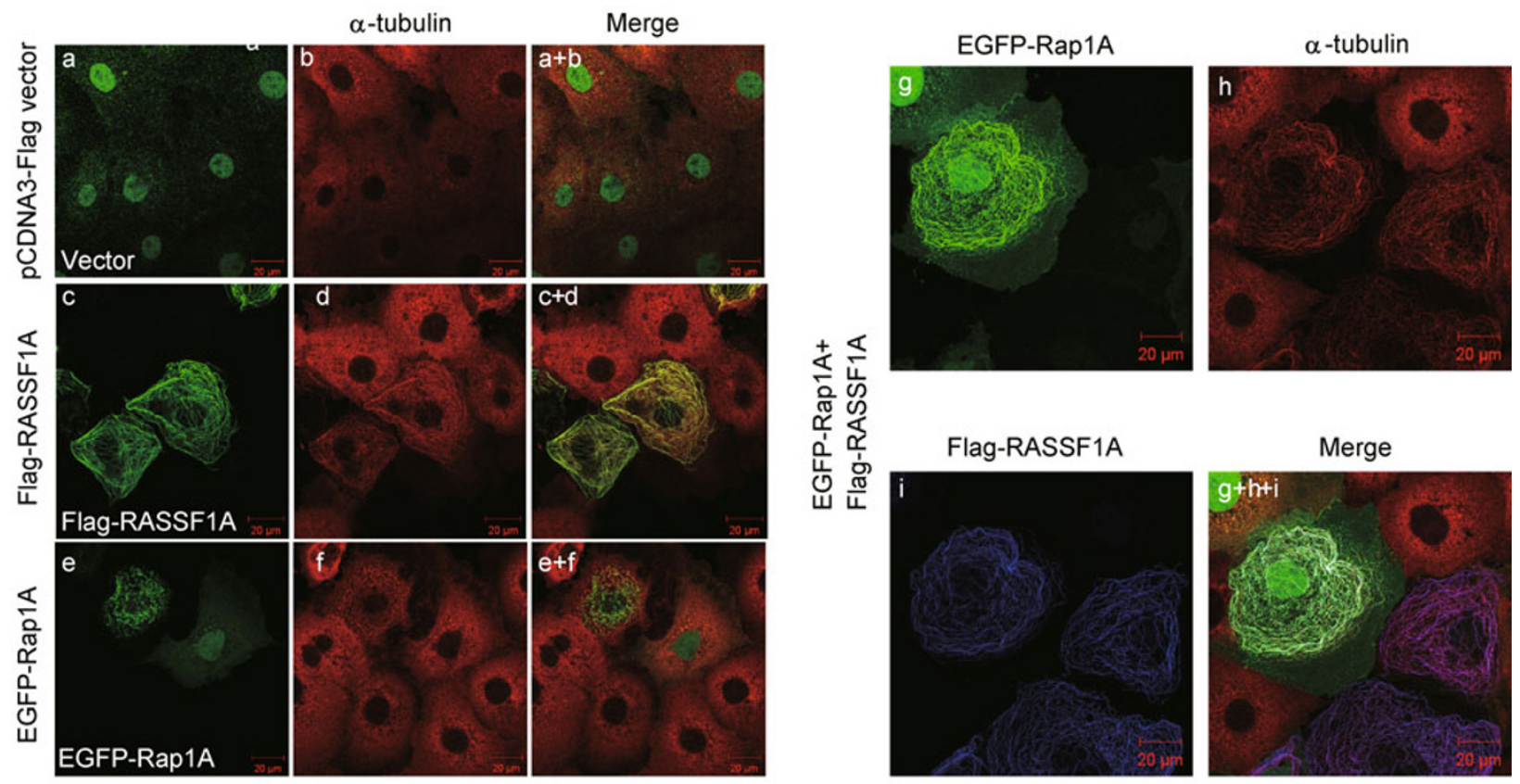

Figure 6. RASSF1A stabilizes microtubules against disruption by nocodazole treatment and the influence of Rap1A. Cos-7 cells transfected with the empty vector $(a, b)$ or the expression vectors encoding Flag-RASSF1A (c, d), EGFP-Rap1A (e, f) or both ( $g$, $\mathrm{h}$, i) were treated with $20 \mu \mathrm{mol} / \mathrm{L}$ nocodazole for $1 \mathrm{~h}$ followed by immunostaining and analysis by confocal microscopy (LSM510, Carl Zeiss Jena). Flag-RASSF1A was visualized by a Flag-M1 antibody and the endogenous microtubule network by an $\alpha$-tubulin antibody. Two-color/multi-color superimposed images (Merge) are shown as indicated. The scale bar is equivalent to $20 \mu \mathrm{m}$.

Chou et al., 2007) and the Golgi (Thyberg and Moskalewski, 1985). To determine whether the RASSF1A + Rap1A induced alteration in microtubule organization influences these processes, the distribution of vimentin and localization of a Golgi marker were assessed. Upon ectopic expression of FlagRASSF1A or co-expression of both the EGFP-Rap1A + FlagRASSF1A in Cos-7 cells, vimentin was found to be retracted in perinuclear bundles (Fig. 7). However, ectopic expression of EGFP-Rap1A alone did not have a visible effect on the distribution pattern of vimentin (data not shown). Similarly, the ectopic expression of Flag-RASSF1A or EGFP-Rap1A led to the partial dispersal of Golgi complex, and their co-expression enhanced this effect resulting in complete fragmentation and dispersal of Golgi complex (Fig. 8). Taken together, these findings suggested that RASSF1A + Rap1A influence the cellular processes that are directly or indirectly dependent on microtubule organization.

\section{DISCUSSION}

Rap1A is a small $G$ protein implicated in an array of biological processes ranging from cell proliferation, differentiation and adhesion to embryogenesis. The effectors through which Rap1A might mediate its diverse functions are largely unknown with the exception of two recent studies correlating Rap1A with integrin-mediated adhesion through RapL (Katagiri et al., 2003) and RIAM (Lafuente et al., 2004). The present study has shown that the tumor suppressor RASSF1A is a candidate novel effector of Rap1A. As typical for most small $G$ proteins, the activation state of Rap1A regulates its interaction with RASSF1A. Additionally, this interaction was also dependent on the phosphorylation state of RASSF1A. Treatment of cells with calyculin A, an inhibitor of the activity of protein phosphatases PP1 and PP2A, led to retardation of the electrophoretic mobility of RASSF1A protein in SDSPAGE, indicative of calyculin A induced phosphorylation of RASSF1A. Furthermore, calyculin A treatment led to inhibition of the in vitro association of RASSF1A with activated Rap1A. The regulation of this interaction is indicative of a functional association.

Intracellular localization of Rap1A and RASSF1A was investigated by confocal microscopy. As reported before, Rap1A was present in perinuclear punctate structures in Cos7 cells (Mochizuki et al., 2001) and RASSF1A was found to co-localize with microtubules (Liu et al., 2003; Dallol et al., 2004; Rong et al., 2004; Song et al., 2004). Ectopically expressed Rap1A or RASSF1A led to rearrangement of the microtubules into circular rings, which appeared to result in the loss of the radial array of microtubules and that of the MTOC. Co-expression of RASSF1A and Rap1A in Cos-7 cells led to robust re-localization of Rap1A from perinuclear punctate structures to circular perinuclear rings where it became strongly co-localized with RASSF1A. This also led to the retraction of microtubules to perinuclear rings, leaving a 

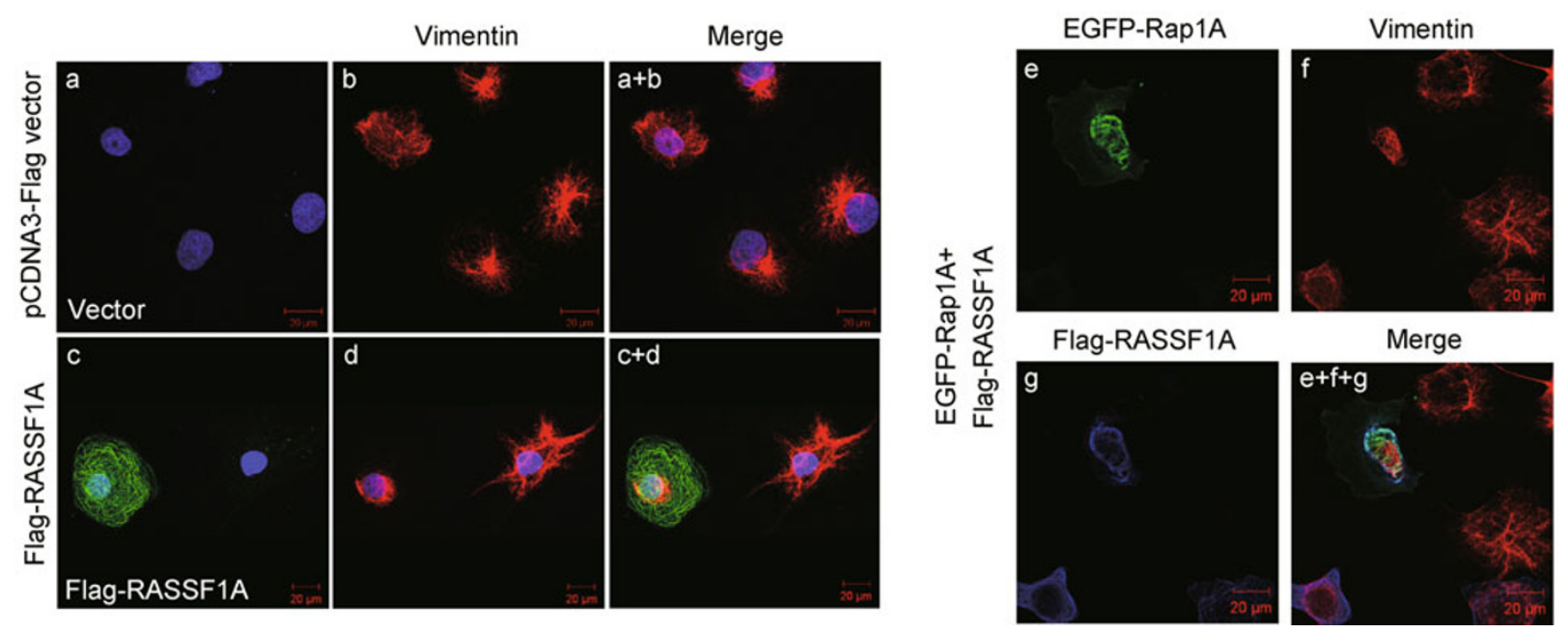

Figure 7. Perinuclear retraction of vimentin upon ectopic expression of Flag-tagged RASSF1A and co-expression of Flag-RASSF1A and EGFP-Rap1A. Cos-7 cells transfected with the empty vector $(a, b)$ or the expression vector encoding FlagRASSF1A (c, d) or Flag-RASSF1A + EGFP-Rap1A (e, f, g) (as indicated in the legend on right side of each panel) were immunostained and analyzed by confocal microscopy (LSM510, Carl Zeiss Jena). Flag-RASSF1A was visualized by a Flag-M1 antibody and vimentin by a vimentin monoclonal (Santa Cruz). Nuclei in $c$ and $d$ were counterstained by DAPI. Multi-color superimposed images (Merge) are shown as indicated. The scale bar is equivalent to $20 \mu \mathrm{m}$.

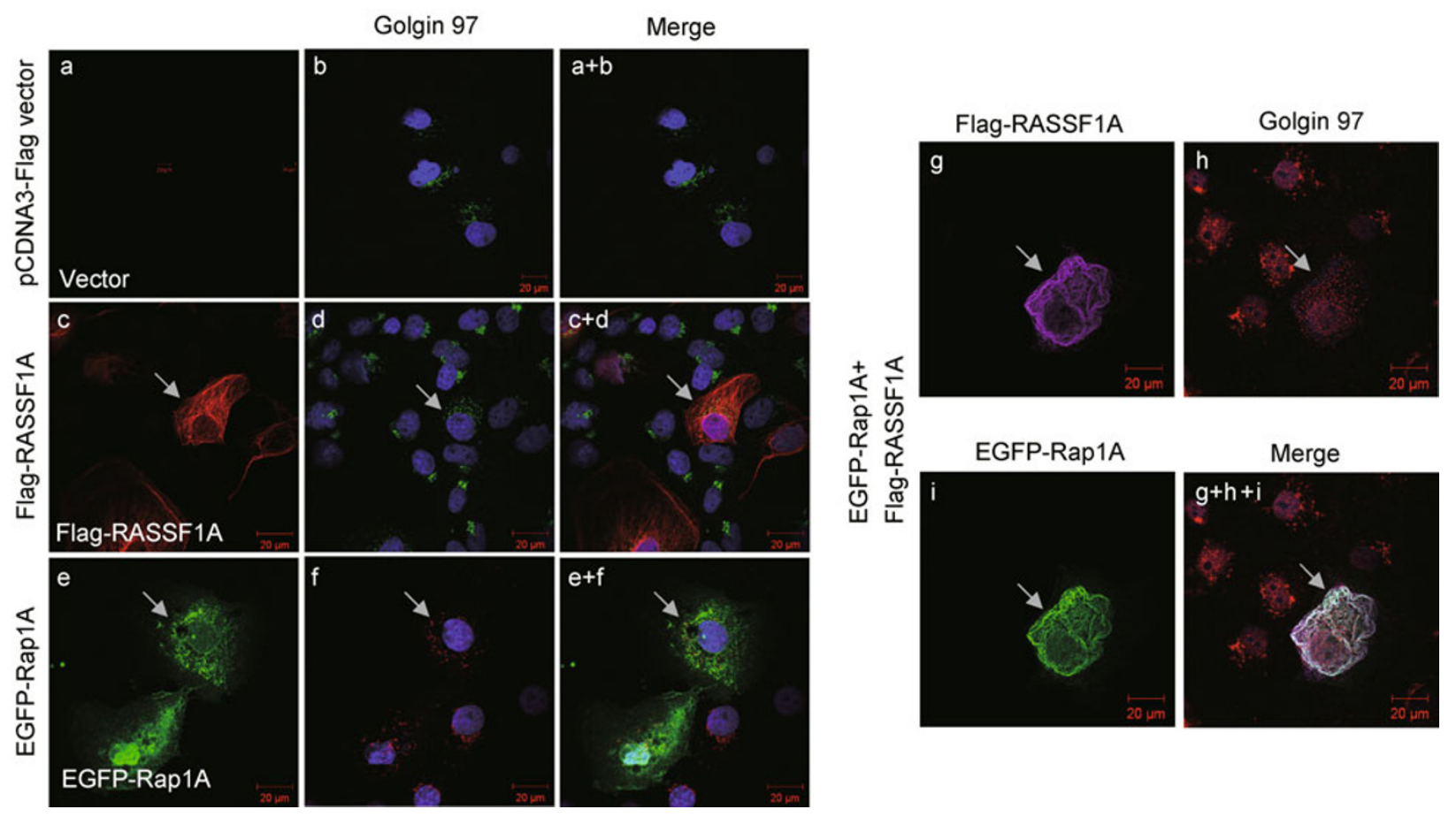

Figure 8. Ectopic expression of Flag-RASSF1A leads to disintegration of the Golgi complex; influence of EGFP-Rap1A. Cos-7 cells transfected with the empty vector $(a, b)$ or the expression vector encoding Flag-RASSF1A (c, d), EGFP-Rap1A (e, f) or both $(\mathrm{g}, \mathrm{h}, \mathrm{i})$ (as indicated in the legend on right side of each panel) were immunostained as indicated and analyzed by confocal microscopy. Flag-RASSF1A was visualized by a Flag-M1 antibody (Rabbit polyclonal, Sigma-Aldrich), and the Golgi complex by the Golgin-97 antibody. Nuclei were counterstained with DAPI with color/multi-color superimposed images (Merge) are shown as indicated. The scale bar is equivalent to $20 \mu \mathrm{m}$. 
substantial part of the cell body devoid of a microtubule network. Thus, it is evident that RASSF1A links Rap1A to microtubules and Rap1A-RASSF1A interaction plays a role in microtubule organization.

The microtubule dependence of the structural organization of Golgi complex has long been established (Robbins and Gonatas, 1964; Thyberg and Moskalewski, 1985, 1999). In view of the fact that ectopic expression of either FlagRASSF1A or EGFP-Rap1A or co-expression of these two has robust effects on intracellular distribution of the microtubule network, the effect of RASSF1A and Rap1A on the integrity of Golgi complex was investigated. It was found that the ectopic expression of Flag-RASSF1A or EGFP-Rap1A led to the partial dispersal of Golgi complex, and the co-expression of these two enhanced this effect resulting in complete fragmentation and dispersal of Golgi complex (Fig. 8).

Several alternative subcellular localizations have been proposed for Rap1A. It is reported that Rap1A is predominantly present at intracellular membranes in the perinuclear region, at endocytic and exocytic vesicles and also at the plasma membrane (Pizon et al., 1994). Rap1A is localized to secretory granules in platelets (Lapetina et al., 1989) and neutrophils (Maridonneau-Parini and de Gunzburg, 1992) and translocates from the granules to the plasma membrane after stimulation of these cells (Maridonneau-Parini and de Gunzburg, 1992; Quinn et al., 1992; D'Silva et al., 1998). In fibroblasts, Rap1A is mainly localized in either the medialGolgi compartment or the early and late endosomes (Béranger et al., 1991; Pizon et al., 1994). There is one report suggesting that an exogenously expressed constitutively active form of Rap1A is localized in the nucleus in $\mathrm{NIH} 3 \mathrm{~T} 3$ fibroblasts and that growth factors stimulate Rap1A translocation from the cytoplasm to the nucleus in squamous carcinoma cells (Mitra et al., 2003). The RASSF1A dependent microtubule recruitment of Rap1A as a perinuclear network has been demonstrated for the first time in this study. Notably, Epac1, which is an exchange factor for Rap1A, has been shown to interact with the microtubule associated protein(s) MAP1A and MAP1B in Cos cells (Gupta and Yarwood, 2005; Borland et al., 2006). MAPs and RASSF1A-assisted physical proximity of Epac1 and Rap1A on microtubules could lead to the rapid onset of mechanisms that lead to Epac1 mediated Rap1A activation and downstream signaling cascade(s).

The influence of Rap1A-RASSF1A on microtubules is particularly notable given the importance of microtubules in many cellular processes including cell division, vesicular traffic, migration and apoptosis (Brinkley, 1997; Mollinedo and Gajate, 2003; Liu et al., 2007). RASSF1A has also been shown to interact with the pro-apoptotic Sterile20-related (Ste20-related) kinase MST1 (mammalian Sterile20-like 1) via its SARAH domain (Khokhlatchev et al., 2002). It is possible that Rap1A-RASSF1A association modulates microtubule behavior through MST1. Interestingly, it has recently been shown that the Rap1A effector RapL, which is related to
RASSF1, can associate with MST1 and regulate the localization and kinase activity of MST1 (Katagiri et al., 2006). In addition, the Drosophila RASSF1A ortholog (dRASSF1) has been shown to restrict the activity of the Drosophila ortholog of MST1 and MST2 (Hpo) by competing with Sav for binding to Hpo (Polesello et al., 2006). It would be intriguing to explore whether the Drosophila Rap1A ortholog (Dras3) also interacts with dRASSF1 and whether this interaction influences the dRASSF1-mediated inhibition of Hpo activity and the microtubule network.

In summary, our present studies have provided valuable information on the selective interaction of the tumor suppressor RASSF1A with Rap1A. The molecular function of this interaction has been examined. It has also been shown that the regulated interaction of Rap1A with RASSF1A plays an important role in microtubule organization.

\section{MATERIALS AND METHODS}

\section{Antibodies and reagents}

Antibodies to Rap1A (rabbit polyclonal) and Vimentin (mouse monoclonal) were from Santa Cruz Biotechnology Inc. (Santa Cruz, CA, USA). Other antibodies used were: Pan-Ras (mouse monoclonal) from Upstate Biotechnology (Upstate, Lake Placid, NY); RASSF1A (mouse monoclonal, ebiosciences, San Diego, CA, USA); $\alpha$-tubulin (mouse monoclonal), $\beta$-tubulin (mouse monoclonal) and Flag-M1 (rabbit polyclonal) from Sigma-Aldrich (Sigma-Aldrich, St. Louis, MO). The antibody Golgin-97 (mouse monoclonal), ProLong ${ }^{\circledR}$ Gold antifade reagent with 4'-6-Diamidino-2-phenylindole (DAPI) and secondary antibodies Alexa Fluor ${ }^{\circledR} 488$ Donkey Anti-Rabbit and Alexa Fluor ${ }^{\circledR} 555$ Donkey Anti-Mouse used for immunofluorescence were from Invitrogen (Invitrogen, Paisley, UK). The Cy5 Donkey AntiRabbit IgG was from Jackson ImmunoResearch Laboratories, Inc. (Jackson ImmunoResearch, Europe). Phospho-RASSF1A S197 and phospho-RASSF1A S203 specific immune sera PPA545 and PPA548, respectively, were produced by us as described elsewhere (Verma et al., 2008). Affinity purified horseradish peroxidase (HRP) coupled mouse and rabbit secondary antibodies, ECL reagent and glutathione Sepharose $4 B$ beads were purchased from GE Healthcare (GE Healthcare, Buckinghamshire, UK). GDP- $\beta-S$, GTP- $\gamma-S$ and Phosphatase inhibitor cocktail 1 and 2 were from Sigma-Aldrich (Sigma-Aldrich, St. Louis, MO). Enzyme T4 DNA ligase, transfection reagent Lipofectamine ${ }^{\mathrm{TM}} \mathbf{2 0 0 0}$ and Zero Blunt TOPO PCR Cloning Kit were from Invitrogen (Invitrogen, Paisley, UK). RapidTrans ${ }^{\mathrm{TM}}$ competent cells used for transformation were purchased from Active Motif (Active Motif, California, USA). All other common reagents were from Sigma-Aldrich (Sigma-Aldrich, St. Louis, MO). The cell culture media were obtained from Cancer Research UK Media Production (CRUK, London, UK).

\section{Plasmid constructs}

The cDNA of the RA/RB domains of RalGDS and Raf, subcloned in pGex4T3 vector (kindly provided by Dr. J. L. Bos, Utrecht University, The Netherlands) (Franke et al., 1997) were used to express RalGDS-RA and Raf-RBD, respectively, as GST-tagged fusions. To 
create a bacterial expression plasmid for Glutathion Sepharose Transferase (GST) fusion protein for Rap1A (GST-Rap1A), the cDNA encoding full-length Rap1A (Human Rap1A, Swiss-Prot accession No. P62834) was PCR amplified from pRK5-HA-Rap1A (a gift from Dr. J. de Gunzburg, Institut Curie-Centre de Recherche and Inserm, Paris) and subcloned into the pGex4T3 bacterial expression vector (GE Healthcare, Buckinghamshire, UK). The constructs pCAGGSEGFP-Rap1A (encoding for amino acid residues 1-184 of Swiss-Prot accession No. P62834), pCAGGS-EGFP-Rap2 (encoding for amino acid residues 1-183 of Swiss-Prot accession No. P10114) and pCAGGS-EGFP-H-Ras (encoding for amino acid residues 1-189 of Swiss-Prot accession No. P01112) were kindly provided by Dr. M. Matsuda (National Cardiovascular Center Research Institute, Japan). To create the bacterial expression plasmid for Glutathion Sepharose Transferase (GST) fusion protein for Rap2 (GST-Rap2) and Ras (GST-Ras), the cDNAs encoding for full-length Rap2 and Ras were PCR amplified from the above vectors (pCAGGS-EGFP-Rap2 and pCAGGS-EGFP-H-Ras, respectively) and subcloned into the pGex4T3 bacterial expression vector (GE Healthcare, Buckinghamshire, UK). pCDNA3-Flag-RASSF1A (encoding for full-length human RASSF1A protein) (Armesilla et al., 2004) was a gift from Dr. L. Neyses (University of Manchester, UK). The plasmid construct pCDNA3-Flag-RASSF1A (S197A-S203A) was created by PCR based site-directed mutagenesis (Stratagene, CA, USA) using pCDNA3-Flag-RASSF1A as a template as described elsewhere (Verma et al., 2008). All the constructs were sequence verified. Plasmid DNA was prepared by standard methods using Qiagen Plasmid Maxi Kits.

\section{Cell culture, transfections and treatments}

HEK293 (human embryonic kidney cells), 293T (human embryonic kidney cell's variant with SV40 Large T-antigen) and green monkey kidney lines Cos-7 (all obtained from CR-UK research services) were routinely maintained in Dulbecco's modified Eagle's medium (DMEM) supplemented with $10 \%$ fetal calf serum (FCS) at $37^{\circ} \mathrm{C}$ in $5 \% \mathrm{CO}_{2}$. Transfections were performed using Lipofectamine 2000 (Invitrogen, Paisley, UK) according to the manufacturer's instructions. The transfections and treatments were performed in six well plates. After stimulation the cells were washed once with ice cold PBS $(137 \mathrm{mmol} / \mathrm{L} \mathrm{NaCl}, 2.7 \mathrm{mmol} / \mathrm{L} \mathrm{KCl}, 4.3 \mathrm{mmol} / \mathrm{L}$ $\mathrm{Na}_{2} \mathrm{HPO}_{4}, 1.47 \mathrm{mmol} / \mathrm{L} \mathrm{KH}_{2} \mathrm{PO}_{4}, \mathrm{pH}$ 7.4) and lysed in $\mathrm{MgCl}_{2}$ containing lysis buffer (MLB) [25 mmol/L HEPES ( $\mathrm{pH} 7.5$ ), $150 \mathrm{mmol} / \mathrm{L} \mathrm{NaCl}, 10 \mathrm{mmol} / \mathrm{L} \mathrm{MgCl}_{2}, 1 \mathrm{mmol} / \mathrm{L}$ EDTA, $10 \%$ glycerol, $1 \%$ Nonidet P-40, $2 \times$ protease inhibitor tablets, $1 \mathrm{mmol} / \mathrm{L}$ phenylmethylsulfonyl fluoride, $0.1 \mu \mathrm{mol} / \mathrm{L}$ aprotinin, $1 \mu \mathrm{mol} / \mathrm{L}$ leupeptin, $1 \mu \mathrm{mol} / \mathrm{L}$ Pepstatin, $1 \mathrm{mmol} / \mathrm{L} \mathrm{Na} \mathrm{NaO}_{4}, 1 \mathrm{mmol} / \mathrm{L} \mathrm{Na} \mathrm{NoO}_{4}$, $4.46 \mathrm{mg} / \mathrm{mL} \mathrm{Na}{ }_{4} \mathrm{P}_{2} \mathrm{O}_{7}, 25 \mathrm{mmol} / \mathrm{L} \beta$-glycerophosphate, $1 \times$ phosphatase inhibitor cocktail 1, $1 \times$ phosphatase inhibitor cocktail 2 (both cocktails from Sigma-Aldrich, St. Louis, MO)]. Cell lysis was performed on ice for $30 \mathrm{~min}$ and the cell lysates were cleared by centrifugation at 13,500 rotation/min for $30 \mathrm{~min}$ at $4^{\circ} \mathrm{C}$ and used immediately for further assays.

\section{Pull-down assay for Rap1A and Ras activation}

Glutathion Sepharose Transferase (GST) fusion proteins (GSTRalGDS-RA and GST-Raf-RBD) (Franke et al., 1997) were purified by standard procedures and the quantity and quality were checked by
SDS-PAGE. Pull-down assays for Rap1 and Ras were performed as described before (Franke et al., 1997).

\section{In vitro loading of Rap1 with GTP-y-S and in vitro Rap1A/Rap2/ Ras binding assays}

The cell lysates containing endogenous Rap1A and/or ectopically expressed EGFP-Rap1A were incubated with $100 \mu \mathrm{mol} / \mathrm{L}$ GTP- $\mathrm{\gamma}-\mathrm{S}$ (or $1 \mathrm{mmol} / \mathrm{L}$ GDP- $\beta-S$ as a negative control) at $30^{\circ} \mathrm{C}$ for $30 \mathrm{~min}$ with agitation to allow the loading of GTP-y-S on Rap1A present in the lysates. Loading was stopped by placing the tubes on ice and adding $60 \mathrm{mmol} / \mathrm{L} \mathrm{MgCl}_{2}$. For Rap1A/Rap2/Ras binding assays bacterial lysates containing approximately $5 \mu \mathrm{g}$ of GST-Rap1A, GST-Rap2 or GST-Ras protein (or GST alone, as a negative control) were incubated with $100 \mu \mathrm{mol} / \mathrm{L}$ GTP-Y-S (or $1 \mathrm{mmol} / \mathrm{L}$ GDP- $\beta$-S as a negative control) at $30^{\circ} \mathrm{C}$ for $30 \mathrm{~min}$ with agitation to allow the loading of GTP-y-S. Loading was stopped by placing the tubes on ice and adding $60 \mathrm{mmol} / \mathrm{L} \mathrm{MgCl}_{2}$. The suspensions were then incubated with glutathione Sepharose $4 \mathrm{~B}$ beads. Following a $1 \mathrm{~h}$ incubation at $4^{\circ} \mathrm{C}$, beads were pelleted and washed three times with ice-cold MLB buffer. These beads were incubated for $90 \mathrm{~min}$ at $4^{\circ} \mathrm{C}$ with cell lysates containing ectopically expressed Flag-tagged RASSF1A. Beads were then washed three times with MLB buffer and subjected to immunoblot (IB) analysis using antibodies to RASSF1A as indicated in the figure legends.

\section{Immunofluorescence}

Cos-7 cells were transfected with plasmids as indicated in the figure legends. $16 \mathrm{~h}$ post-transfection the cells were trypsinized and seeded at $60 \%$ confluence on acid washed glass coverslips. Cells were allowed to grow for an additional $16 \mathrm{~h}$. The cells were washed in PBS, fixed with $3.7 \%$ paraformaldehyde for $10 \mathrm{~min}$ and permeabilized with $0.1 \%$ Triton $X-100$ for 5 min. The coverslips were then treated with $1 \%$ BSA in PBS for $30 \mathrm{~min}$ at RT followed by incubation with an appropriate dilution of primary antibody prepared in 1\% BSA. After washing in PBS, the coverslips were incubated in the dark with fluorescent dye-conjugated secondary antibody for $45 \mathrm{~min}$ at RT. Finally, the coverslips were washed in $3 \times$ PBS and $1 \times$ water and mounted onto glass slides using ProLong ${ }^{\circledR}$ Gold antifade reagent with DAPI (Invitrogen, Paisley, UK). The slides were left at RT overnight to allow the mountant to set and the images were acquired using an upright laser scanning confocal microscope (LSM 510 Carl Zeiss Jena) equipped with a $64 \times / 1.4$ Plan-Apochromat Oil Ph3 objective. EGFP and Alexa Fluor ${ }^{\circledR} 488$ were excited with the $488 \mathrm{~nm}$ line of an Argon laser, Alexa Fluor ${ }^{\circledR} 555$ with the $543 \mathrm{~nm}$ line and Cy 5 with the $633 \mathrm{~nm}$ line in HeNe laser. Each image represents a single $0.38 \mu \mathrm{m}$ ' $Z$ ' optical section and each confocal image is representative of at least four separate experiments.

\section{ACKNOWLEDGEMENTS}

We thank Drs. J. L. Bos, Utrecht University, The Netherlands; J. de Gunzburg, Institut Curie-Centre de Recherche and Inserm, Paris; M. Matsuda, National Cardiovascular Center Research Institute, Japan and L. Neyses, University of Manchester, UK for generously providing reagents. SKV is extremely grateful to Dr. Valentine M. Macaulay, CRUK Molecular Oncology Laboratories, Weatherall Institute of Molecular Medicine, Oxford, UK for constant encouragement and critical 
appraisal during the course of this study. SKV also acknowledges the award of the Commonwealth Scholarship and the financial support received from the Department of Medical Oncology, Medical Sciences Division, The University of Oxford, Oxford, UK and the Cancer Research UK.

Supplementary material is available in the online version of this article at http://dx.doi.org/10.1..7/s13238-011-1028-z and is accessible for authorized users.

\section{ABBREVIATIONS}

GST, Glutathion Sepharose Transferase; GST-Rap1A, Glutathion Sepharose Transferase (GST) fusion protein for Rap1A; IB, immunoblot; MTOC, microtubule-organizing centre; NJ, neighbour joining; RASSF1A, Ras association domain family 1A; RA, Rasassociation

\section{REFERENCES}

Armesilla, A.L., Williams, J.C., Buch, M.H., Pickard, A., Emerson, M., Cartwright, E.J., Oceandy, D., Vos, M.D., Gillies, S., Clark, G.J., et al. (2004). Novel functional interaction between the plasma membrane $\mathrm{Ca} 2+$ pump $4 \mathrm{~b}$ and the proapoptotic tumor suppressor Ras-associated factor 1 (RASSF1). J Biol Chem 279, 31318-31328.

Asha, H., de Ruiter, N.D., Wang, M.G., and Hariharan, I.K. (1999). The Rap1 GTPase functions as a regulator of morphogenesis in vivo. EMBO J 18, 605-615.

Bailly, E., and Bornens, M. (1992). Cell biology. Centrosome and cell division. Nature 355, 300-301.

Béranger, F., Goud, B., Tavitian, A., and de Gunzburg, J. (1991). Association of the Ras-antagonistic Rap $1 / \mathrm{Krev}-1$ proteins with the Golgi complex. Proc Natl Acad Sci U S A 88, 1606-1610.

Boettner, B., Govek, E.E., Cross, J., and Van Aelst, L. (2000). The junctional multidomain protein AF-6 is a binding partner of the Rap1A GTPase and associates with the actin cytoskeletal regulator profilin. Proc Natl Acad Sci U S A 97, 9064-9069.

Borland, G., Gupta, M., Magiera, M.M., Rundell, C.J., Fuld, S., and Yarwood, S.J. (2006). Microtubule-associated protein 1B-light chain 1 enhances activation of Rap1 by exchange protein activated by cyclic AMP but not intracellular targeting. Mol Pharmacol 69, 374-384.

Bos, J.L., de Rooij, J., and Reedquist, K.A. (2001). Rap1 signalling: adhering to new models. Nat Rev Mol Cell Biol 2, 369-377.

Brinkley, W. (1997). Microtubules: a brief historical perspective. J Struct Biol 118, 84-86

Burney, T.L., Rockove, S., Eiseman, J.L., Jacobs, S.C., and Kyprianou, N. (1994). Partial growth suppression of human prostate cancer cells by the Krev-1 suppressor gene. Prostate 25, 177-188.

Chou, Y.H., Flitney, F.W., Chang, L., Mendez, M., Grin, B., and Goldman, R.D. (2007). The motility and dynamic properties of intermediate filaments and their constituent proteins. Exp Cell Res 313, 2236-2243.

D'Silva, N.J., Jacobson, K.L., Ott, S.M., and Watson, E.L. (1998). Beta-adrenergic-induced cytosolic redistribution of Rap1 in rat parotid acini: role in secretion. Am J Physiol 274, C1667-C1673.
Dallol, A., Agathanggelou, A., Fenton, S.L., Ahmed-Choudhury, J., Hesson, L., Vos, M.D., Clark, G.J., Downward, J., Maher, E.R., and Latif, F. (2004). RASSF1A interacts with microtubule- associated proteins and modulates microtubule dynamics. Cancer Res 64, 4112-4116.

Damak, S., Harnboonsong, Y., George, P.M., and Bullock, D.W. (1996). Expression of human Krev-1 gene in lungs of transgenic mice and subsequent reduction in multiplicity of ethyl carbamateinduced lung adenomas. Mol Carcinog 17, 84-91.

Dammann, R., Li, C., Yoon, J.H., Chin, P.L., Bates, S., and Pfeifer, G. P. (2000). Epigenetic inactivation of a RAS association domain family protein from the lung tumor suppressor locus $3 p 21.3$. Nat Genet 25, 315-319.

Dammann, R., Schagdarsurengin, U., Seidel, C., Strunnikova, M., Rastetter, M., Baier, K., and Pfeifer, G.P. (2005). The tumor suppressor RASSF1A in human carcinogenesis: an update. Histol Histopathol 20, 645-663.

Downing, K.H. (2000). Structural basis for the interaction of tubulin with proteins and drugs that affect microtubule dynamics. Annu Rev Cell Dev Biol 16, 89-111.

Franke, B., Akkerman, J.W., and Bos, J.L. (1997). Rapid Ca2 +mediated activation of Rap1 in human platelets. EMBO J 16, 252-259.

Gupta, M., and Yarwood, S.J. (2005). MAP1A light chain 2 interacts with exchange protein activated by cyclic AMP 1 (EPAC1) to enhance Rap1 GTPase activity and cell adhesion. J Biol Chem 280, 8109-8116.

Hariharan, I.K., Carthew, R.W., and Rubin, G.M. (1991). The Drosophila roughened mutation: activation of a rap homolog disrupts eye development and interferes with cell determination. Cell 67, 717-722.

Herrmann, C., Horn, G., Spaargaren, M., and Wittinghofer, A. (1996). Differential interaction of the ras family GTP-binding proteins $\mathrm{H}$ Ras, Rap1A, and R-Ras with the putative effector molecules Raf kinase and Ral-guanine nucleotide exchange factor. J Biol Chem 271, 6794-6800.

Hogue, C.W. (1997). Cn3D: a new generation of three-dimensional molecular structure viewer. Trends Biochem Sci 22, 314-316.

Huang, L., Weng, X., Hofer, F., Martin, G.S., and Kim, S.H. (1997). Three-dimensional structure of the Ras-interacting domain of RalGDS. Nat Struct Biol 4, 609-615.

Jelinek, M.A., and Hassell, J.A. (1992). Reversion of middle T antigen-transformed Rat-2 cells by Krev-1: implications for the role of p21c-ras in polyomavirus-mediated transformation. Oncogene 7, 1687-1698.

Katagiri, K., Imamura, M., and Kinashi, T. (2006). Spatiotemporal regulation of the kinase Mst1 by binding protein RAPL is critical for lymphocyte polarity and adhesion. Nat Immunol 7, 919-928.

Katagiri, K., Maeda, A., Shimonaka, M., and Kinashi, T. (2003). RAPL, a Rap1-binding molecule that mediates Rap1-induced adhesion through spatial regulation of LFA-1. Nat Immunol 4, 741-748.

Khokhlatchev, A., Rabizadeh, S., Xavier, R., Nedwidek, M., Chen, T., Zhang, X.F., Seed, B., and Avruch, J. (2002). Identification of a novel Ras-regulated proapoptotic pathway. Curr Biol 12, 253265.

Kitayama, H., Sugimoto, Y., Matsuzaki, T., Ikawa, Y., and Noda, M. (1989). A ras-related gene with transformation suppressor activity. 
Cell 56, 77-84.

Koradi, R., Billeter, M., and Wuthrich, K. (1996). MOLMOL: a program for display and analysis of macromolecular structures. J Mol Graph 14, 51-55, 29-32.

Lafuente, E.M., van Puijenbroek, A.A., Krause, M., Carman, C.V., Freeman, G.J., Berezovskaya, A., Constantine, E., Springer, T.A., Gertler, F.B., and Boussiotis, V.A. (2004). RIAM, an Ena/VASP and Profilin ligand, interacts with Rap1-GTP and mediates Rap1induced adhesion. Dev Cell 7, 585-595.

Lapetina, E.G., Lacal, J.C., Reep, B.R., and Molina y Vedia, L. (1989). $A$ ras-related protein is phosphorylated and translocated by agonists that increase cAMP levels in human platelets. Proc Natl Acad Sci U S A 86, 3131-3134.

Leach, S.D., Berger, D.H., Davidson, B.S., Curley, S.A., and Tainsky, M.A. (1998). Enhanced Krev-1 expression inhibits the growth of pancreatic adenocarcinoma cells. Pancreas 16, 491-498.

Lerman, M.I., and Minna, J.D. (2000). The 630-kb lung cancer homozygous deletion region on human chromosome 3 p21.3: identification and evaluation of the resident candidate tumor suppressor genes. The International Lung Cancer Chromosome 3p21.3 Tumor Suppressor Gene Consortium. Cancer Res 60, 6116-6133.

Liao, G., and Gundersen, G.G. (1998). Kinesin is a candidate for cross-bridging microtubules and intermediate filaments. Selective binding of kinesin to detyrosinated tubulin and vimentin. J Biol Chem 273, 9797-9803.

Liu, L., Tommasi, S., Lee, D.H., Dammann, R., and Pfeifer, G.P. (2003). Control of microtubule stability by the RASSF1A tumor suppressor. Oncogene 22, 8125-8136.

Liu, Z., Vong, Q.P., and Zheng, Y. (2007). CLASPing microtubules at the trans-Golgi network. Dev Cell 12, 839-840.

Maridonneau-Parini, I., and de Gunzburg, J. (1992). Association of rap1 and rap2 proteins with the specific granules of human neutrophils. Translocation to the plasma membrane during cell activation. J Biol Chem 267, 6396-6402.

Mitra, R.S., Zhang, Z., Henson, B.S., Kurnit, D.M., Carey, T.E., and D'Silva, N.J. (2003). Rap1A and rap1B ras-family proteins are prominently expressed in the nucleus of squamous carcinomas: nuclear translocation of GTP-bound active form. Oncogene 22, 6243-6256.

Mochizuki, N., Yamashita, S., Kurokawa, K., Ohba, Y., Nagai, T., Miyawaki, A., and Matsuda, M. (2001). Spatio-temporal images of growth-factor-induced activation of Ras and Rap1. Nature 411, 1065-1068.

Mollinedo, F., and Gajate, C. (2003). Microtubules, microtubuleinterfering agents and apoptosis. Apoptosis 8, 413-450.

Nassar, N., Horn, G., Herrmann, C., Block, C., Janknecht, R., and Wittinghofer, A. (1996). Ras/Rap effector specificity determined by charge reversal. Nat Struct Biol 3, 723-729.

Nassar, N., Horn, G., Herrmann, C., Scherer, A., McCormick, F., and Wittinghofer, A. (1995). The 2.2 A crystal structure of the Rasbinding domain of the serine/threonine kinase c-Raf1 in complex with Rap1A and a GTP analogue. Nature 375, 554-560.

Pizon, V., Desjardins, M., Bucci, C., Parton, R.G., and Zerial, M. (1994). Association of Rap1a and Rap1b proteins with late endocytic/phagocytic compartments and Rap2a with the Golgi complex. J Cell Sci 107, 1661-1670.
Polesello, C., Huelsmann, S., Brown, N.H., and Tapon, N. (2006). The Drosophila RASSF homolog antagonizes the hippo pathway. Curr Biol 16, 2459-2465.

Prahlad, V., Yoon, M., Moir, R.D., Vale, R.D., and Goldman, R.D. (1998). Rapid movements of vimentin on microtubule tracks: kinesin-dependent assembly of intermediate filament networks. J Cell Biol 143, 159-170.

Quinn, M.T., Mullen, M.L., Jesaitis, A.J., and Linner, J.G. (1992). Subcellular distribution of the Rap1A protein in human neutrophils: colocalization and cotranslocation with cytochrome b559. Blood 79, 1563-1573.

Robbins, E., and Gonatas, N.K. (1964). The Ultrastructure of a Mammalian Cell During the Mitotic Cycle. J Cell Biol 21, 429-463.

Rong, R., Jin, W., Zhang, J., Sheikh, M.S., and Huang, Y. (2004). Tumor suppressor RASSF1A is a microtubule-binding protein that stabilizes microtubules and induces G2/M arrest. Oncogene 23, 8216-8230.

Sato, K.Y., Polakis, P.G., Haubruck, H., Fasching, C.L., McCormick, F., and Stanbridge, E.J. (1994). Analysis of the tumor suppressor activity of the K-rev-1 gene in human tumor cell lines. Cancer Res 54, 552-559.

Sekido, Y., Ahmadian, M., Wistuba, I.I., Latif, F., Bader, S., Wei, M.H., Duh, F.M., Gazdar, A.F., Lerman, M.I., and Minna, J.D. (1998). Cloning of a breast cancer homozygous deletion junction narrows the region of search for a 3 p21.3 tumor suppressor gene. Oncogene 16, 3151-3157.

Shindyalov, I.N., and Bourne, P.E. (1998). Protein structure alignment by incremental combinatorial extension (CE) of the optimal path. Protein Eng 11, 739-747.

Shivakumar, L., Minna, J., Sakamaki, T., Pestell, R., and White, M.A. (2002). The RASSF1A tumor suppressor blocks cell cycle progression and inhibits cyclin D1 accumulation. Mol Cell Biol 22, 4309-4318.

Song, M.S., Song, S.J., Ayad, N.G., Chang, J.S., Lee, J.H., Hong, H. K., Lee, H., Choi, N., Kim, J., Kim, H., et al. (2004). The tumor suppressor RASSF1A regulates mitosis by inhibiting the APCCdc20 complex. Nat Cell Biol 6, 129-137.

Thyberg, J., and Moskalewski, S. (1985). Microtubules and the organization of the Golgi complex. Exp Cell Res 159, 1-16.

Thyberg, J., and Moskalewski, S. (1999). Role of microtubules in the organization of the Golgi complex. Exp Cell Res 246, 263-279.

Tommasi, S., Dammann, R., Zhang, Z., Wang, Y., Liu, L., Tsark, W.M., Wilczynski, S.P., Li, J., You, M., and Pfeifer, G.P. (2005). Tumor susceptibility of Rassf1a knockout mice. Cancer Res 65, 92-98.

Verma, S.K., Ganesan, T.S., and Parker, P.J. (2008). The tumor suppressor RASSF1A is a novel substrate of PKC. FEBS Lett 582, 2270-2276.

Vetter, I.R., Linnemann, T., Wohlgemuth, S., Geyer, M., Kalbitzer, H. R., Herrmann, C., and Wittinghofer, A. (1999). Structural and biochemical analysis of Ras-effector signaling via RalGDS. FEBS Lett 451, 175-180.

Vos, M.D., Ellis, C.A., Bell, A., Birrer, M.J., and Clark, G.J. (2000). Ras uses the novel tumor suppressor RASSF1 as an effector to mediate apoptosis. J Biol Chem 275, 35669-35672.

Zhang, Z., Rehmann, H., Price, L.S., Riedl, J., and Bos, J.L. (2005). AF6 negatively regulates Rap1-induced cell adhesion. J Biol Chem 280, 33200-33205. 